\title{
COMMISSIONING OF THE LNLS ELLIPTICALLY POLARIZING UNDULATOR
}

\author{
J. Citadini, N. P. Abreu, R. H. A. Farias, M. J. Ferreira, J. G. R. S. Franco, A. F. Gouveia, \\ L. C. Jahnel, L. Liu, X. R. Resende, P. F. Tavares, G. Tosin, LNLS, Campinas, Brazil
}

\begin{abstract}
We present the results of the commissioning of the first Elliptically Polarizing Undulator (EPU) to be installed at the $1.37 \mathrm{GeV}$ electron storage ring of the Brazilian Synchrotron Light Source (LNLS). The undulator is designed to provide UV and soft X-ray photons from $100 \mathrm{eV}$ up to $1 \mathrm{keV}$ with full polarization control. It uses the APPLE II design and allows for both parallel and antiparallel longitudinal motion of its magnet cassettes. We present the commissioning results including the measured orbit and tune perturbations of the undulator fields on the stored beam.
\end{abstract}

\section{INTRODUCTION}

The LNLS UVX synchrotron light source has been in operation for users since 1997. The now 10-year-old light source is based on a $1.37 \mathrm{GeV}$ electron storage ring and had its first insertion device, a $2 \mathrm{~T}$ multipolar wiggler, installed and commissioned 2 years ago. The ring is $93 \mathrm{~m}$ long and its magnetic lattice has 6-fold symmetry with four straight sections, each one suitable for the installation of insertion devices up to $2.9 \mathrm{~m}$ long. The other two sections house the two RF cavities and the injection septa. The injection system to the storage ring comprises a small linac and a $500 \mathrm{MeV}$ booster synchrotron. In the multibunch operation mode the typical initial stored beam current in user runs is $250 \mathrm{~mA}$.

There are currently 14 operational beam lines open for users. Except for the wiggler based Protein Crystallography beam line, optimized for the Multiple Wavelength Anomalous Dispersion (MAD) technique, all the others are bending magnet based $(1.67 \mathrm{~T}$ at $1.37 \mathrm{GeV})$ with critical photon energy of $2 \mathrm{keV}$. Three of these beam

\begin{tabular}{|c|c|c|}
\hline \multicolumn{3}{|l|}{ Basic Parameters } \\
\hline Type & Apple-II & \\
\hline $\begin{array}{l}\text { Max Peak Fields (vertical, } \\
\text { circular and horizontal phases) }\end{array}$ & $\begin{array}{l}0.53,0,27 \\
\text { and } 0.31\end{array}$ & $\mathrm{~T}$ \\
\hline Period & 50 & $\mathrm{~mm}$ \\
\hline Number of periods & 54 & \\
\hline Minimum Gap & 22 & $\mathrm{~mm}$ \\
\hline Maximum Gap & 300 & $\mathrm{~mm}$ \\
\hline Length & 2.773 & $\mathrm{~m}$ \\
\hline $\begin{array}{l}\text { Horizontal distance between } \\
\text { cassettes }\end{array}$ & 1.3 & $\mathrm{~mm}$ \\
\hline Magnet Material & $\mathrm{NdFeB}$ & \\
\hline $\begin{array}{l}\text { Total radiated power @ } 250 \mathrm{~mA} \text {, } \\
\text { minimum gap, } 0^{\circ} \text { phase }\end{array}$ & 285 & $\mathrm{~W}$ \\
\hline
\end{tabular}

Table 1: Main Undulator Parameters lines operate in the ultra-violet region of the spectrum. The second insertion device installed in the light source is an elliptically polarizing undulator (EPU), optimized to produce radiation in the UV and soft X-ray range with complete control of polarization. The device was installed last March and is now under commissioning.

In this paper we present an overview of the main characteristics of the EPU and some comments about its installation in the storage ring. We present the results of the commissioning of the new device in the storage ring, focussing on the effects on the machine optics.

\section{THE UNDULATOR}

The new EPU device was designed and constructed at the LNLS [1]. It uses a pure permanent magnet design. The magnetic structure is composed of two pairs of magnet linear arrays or cassettes. Each magnetic period contains four blocks in Halbach configuration. The field intensity is controlled by changing the gap aperture (gap motion) and the light polarization is changed by the simultaneous displacement of two diagonally placed cassettes (phase motion). The mechanical structure is composed of a C-Frame, with gap and phase actuators with absolute encoders and biased with springs to eliminate backlash. Each one of the four magnetic blocks linear arrays is segmented into seven sub-cassettes. Field correction is carried out by means of virtual shims, with horizontal and vertical displacements.

The device is designed to cover the photon flux in the range from $100 \mathrm{eV}$ to $1000 \mathrm{eV}$ (124 $\AA$ to $12.4 \AA$ ), allowing linear, elliptical and circular polarizations. The main parameters of the EPU are listed on Table 1.

Careful magnetic measurements and fine tuning of the multipole components of the magnetic field of the undulator were performed in order to meet the specifications [2]. Detailed characterization of each individual block of permanent magnet supplied input data for sorting algorithms used to arrange the magnets to minimize the multipole field integrals, which combined with the use of virtual shims brought the magnetic field of the undulator close to the specifications. L-shims were applied close to the end poles in order to bring down multipole field components at phase $\pm 25 \mathrm{~mm}$.

Field quality constraints required by dynamic aperture calculations led to the installation of a set of built-in coils, at both ends of the undulator, designed for skew multipole field compensation. The role of these coils is to reduce the impact of the phase motion on the vertical coupling of the beam, on dynamic aperture and beam lifetime. 


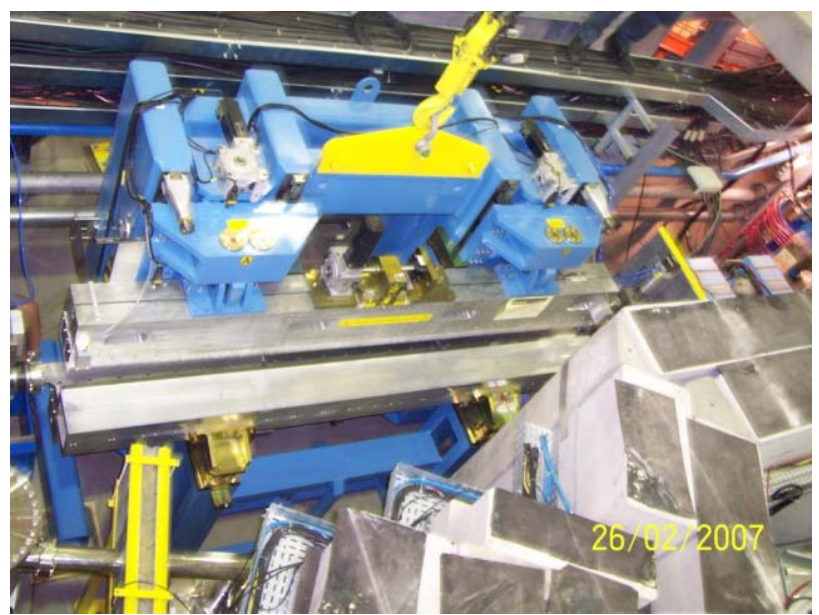

Figure 1: Installation of the EPU in the storage ring.

Measurements performed with the electron beam show good agreement between the observed perturbations and the integrated field bench measurements.

\section{Steering coils}

In addition to the compensation coils two sets of steering coils for horizontal and vertical local orbit correction were installed at both sides of the undulator. These coils are driven by independent power supplies and are installed in the storage ring girders. The coils are designed to permit fast local orbit correction during the operation of the EPU.

Two additional RF BPMs have been installed at both sides of the EPU in order to allow for improved orbit control at the undulator straight section.

\section{Vacuum chamber}

The vacuum chamber is an improvement on the design of the chamber built for the wiggler. It is a $3 \mathrm{~m}$ long, $1.2 \mathrm{~mm}$ thick $316 \mathrm{~L}$ SS tube, which was mechanically pressed into an elliptical shape from an originally round tube. The vertical beam stay clear is $18.6 \mathrm{~mm}$. In order to accomplish the required mechanical tolerances, the tube is welded to suitably machined lateral supports all along the whole length of the chamber. The chamber matched both the mechanical and magnetic specifications and had its inner surface NEG-coated in order to minimize the impact of the vacuum intervention in the machine.

\section{INSTALLATION AND COMMISSIONING}

In order to minimize the effect of the undulator on the optical properties of the storage ring a special low vertical betatron mode at the undulator section was established and is now the standard operational mode during users run. As expected from the results of the magnetic bench characterization, the effect of closing the gap of the EPU on beam orbit and tunes is very small. There have been no setbacks in the commissioning of the control system and in running the machine with the new device.

Effects of the EPU on dynamic aperture could not be detected from lifetime measurements even with the skew multipole compensation coils powered off. No systematic 07 Accelerator Technology Main Systems

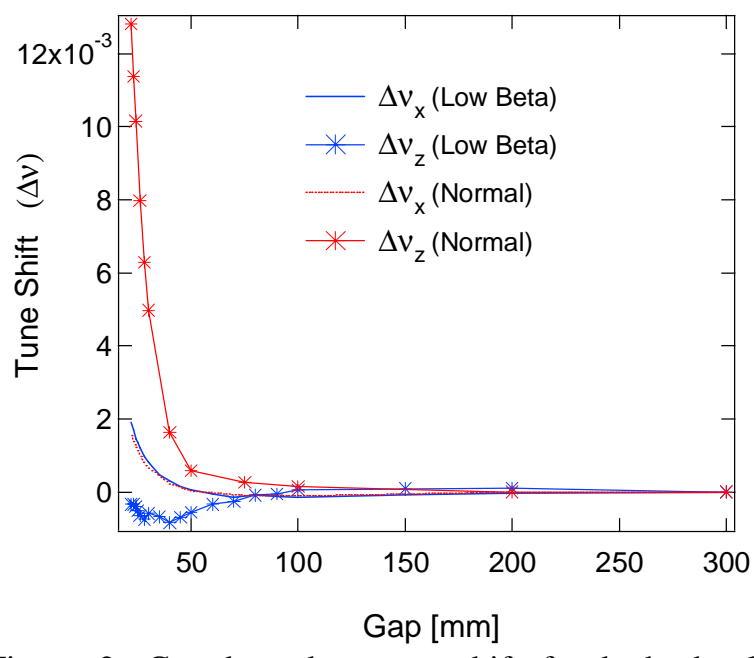

Figure 2: Gap-dependent tune shift for both the low vertical betatron mode and for the normal mode.

variation in lifetime was detected at any EPU setting for the low vertical betatron operation mode. The analysis of the effect of the EPU on the machine has up to now been focussed on the linear optics aspects.

\section{Tune measurements}

Insertion devices introduce mainly vertical tune shifts on the circulating beam due to their edge focussing effects. Tune shifts of the EPU have been measured as a function of the gap [Fig.2] and as a function of the row phase [Fig.3]. For the low betatron mode the tune shifts are quite small. In this mode it is possible to operate the undulador without any tune correction mechanism.

For comparison, in [Fig.3] curves of tune shifts calculated from perturbation theory are plotted. A perfect sinusoidal field was taken in the modelling since it has all the important symmetries of the EPU field and the perturbation theory is correct up to second order in the small parameters $\theta_{\mathrm{x}}$ and $\theta_{\mathrm{z}}$ (maximum horizontal and vertical angle deflections):

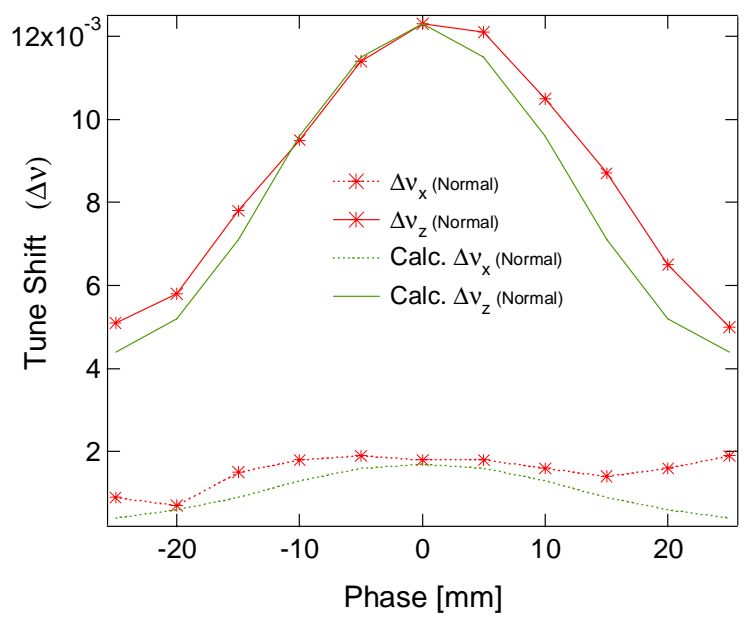

Figure 3: Measured phase-dependent tune shift through a complete period of $\pm 25 \mathrm{~mm}$. Calculated tune shifts from a model field for the normal mode are plotted for comparison.

T09 Room-Temperature Magnets 


$$
\theta_{x}=\frac{e c}{2 \pi E} B_{v}(g) \cos \left(\pi \frac{P}{\lambda}\right) ; \quad \theta_{z}=\frac{e c}{2 \pi E} B_{h}(g) \sin \left(\pi \frac{P}{\lambda}\right)
$$

Here, $e$ is the elementary charge, $c$ is the speed of light, $E$ is the beam energy, $\lambda$ is the undulator period and $P$ its row phase. $\mathrm{B}_{\mathrm{h}}(\mathrm{g})$ and $\mathrm{B}_{\mathrm{v}}(\mathrm{g})$ are the gap dependent horizontal and vertical peak fields, respectively. Within perturbation theory, tune shifts are given by the expression [3]

$$
\begin{aligned}
& \delta v_{x}=-\frac{L / \lambda}{8 \pi} G\left(\beta_{x}\right)\left(k_{v}^{2} \theta_{x}^{2}+k_{h}^{2} \theta_{z}^{2}\right) \\
& \delta v_{z}=\frac{L / \lambda}{8 \pi} G\left(\beta_{z}\right)\left(\left(4 \pi^{2}+k_{v}^{2}\right) \theta_{x}^{2}+\left(4 \pi^{2}+k_{h}^{2}\right) \theta_{z}^{2}\right)
\end{aligned}
$$

where $\mathrm{G}(\beta)=\beta / \lambda+(\lambda / \beta)\left(2+(\mathrm{L} / \lambda)^{2}\right) / 12, \mathrm{~L}$ is the EPU length, $\beta_{x, z}$ are the unperturbed betatron functions at the center of the undulator straight section, and $\mathrm{k}_{\mathrm{v}}{ }^{2}=-\left(\lambda^{2} / \mathrm{B}_{\mathrm{v}}\right) \mathrm{d}^{2} \mathrm{~B}_{\mathrm{v}} /\left.\mathrm{dx}^{2}\right|_{0}$ and $\mathrm{k}_{\mathrm{h}}{ }^{2}=-\left(\lambda^{2} / \mathrm{B}_{\mathrm{h}}\right) \mathrm{d}^{2} \mathrm{~B}_{\mathrm{h}} /\left.\mathrm{dx}^{2}\right|_{0}$ are the transverse roll-offs of the horizontal and vertical field components.

The agreement between the model and measured tune shifts is good for the normal mode [Fig.3]. On the other hand, there was no set of good fitting parameters $\mathrm{k}_{\mathrm{h}, \mathrm{v}}{ }^{2}$ for the low beta mode. One possible explanation is that, for this special mode with low vertical beta at the center and large beta at the ends of the EPU, the effect of the end fields - which is not correctly modelled in the calculation - is comparable to the small tune shift contribution of the central part of undulator.

\section{Beam Size}

There is a dedicated e-beam diagnostics beamline at the LNLS storage ring with a pinhole grid and an X-ray camera for beam imaging [4]. Beam size, angle and centroid position were measured as functions of the EPU gap and phase using this imaging setup. Apart from an increase of the vertical beam size, no systematic changes were observed on other parameters when the gap was closed. The measured vertical beam size increase of $\sim 25$ $\mu \mathrm{m}$ at the diagnostics beamline [Fig.4] scales to an increase of $5 \mu \mathrm{m}$ and $12 \mu \mathrm{m}$ respectively at the undulator centre and ends. This increase in size is expected to be acceptable in terms of radiation quality degradation.

\section{Orbit distortion}

Orbit distortions produced by the EPU are too large compared to what is acceptable during typical user runs. The acceptable maximum is $\pm 5 \mu \mathrm{m}$ off the reference orbit at the BPMs during EPU operations.

Measurements have been performed to determine the orbit distortions caused by the undulator as a function of its gap and phase settings. With these data a table with the local steering coil strengths were calculated to compensate the distortions. We expect to operate user runs with feed-forward orbit correction based on this table. The final table was determined after two iterations of orbit correction. This was necessary since residual coupling in the ring causes a residual horizontal orbit

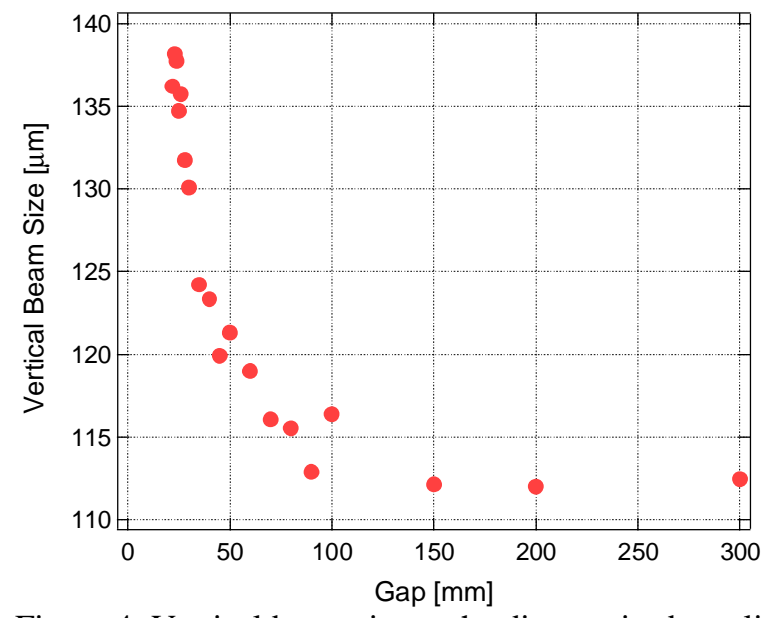

Figure 4: Vertical beam size at the diagnostics beamline.

distortion when vertical correction is applied and viceversa. In fact, distortions on the order of $100 \mu \mathrm{m}$ without correction are reduced to about $10 \mu \mathrm{m}$ after one correction iteration and to $2-3 \mu \mathrm{m}$ after two iterations. This value is already acceptable for user runs.

During data analysis for parallel row motion we found that the final table can also be obtained in just one iteration if coupling effects are explicitly considered in the beam response function. Actually, the table for antiparallel row motion was obtained in just one iteration.

The values for the residual orbit after correction using the feed-forward table were obtained for static measurements, i.e., the orbit was measured after the EPU completely stopped its gap or phase motion. During the movement, orbit distortion can be higher depending on the gap/phase changing velocity. At present the maximum acceptable velocity is limited by the control system response time.

\section{SUMMARY}

The commissioning of the LNLS EPU is under way. Preliminary results show that the effects of the undulator on the machine performance are small and that orbit distortions can be kept inside acceptable values if movements are very slow $(<10 \mathrm{~mm} / \mathrm{min})$. Efforts are now focussed on adapting the orbit compensation system to allow faster EPU operations and on investigating the nonlinear effects of the EPU on the machine optics.

\section{REFERENCES}

[1] G Tosin et al., "Conceptual Design of an EPU for VUV radiation production at LNLS", EPAC'06, Edinburgh, UK, June 2006.

[2] G Tosin et al., "Final Adjustment of the Magnetic Field of the LNLS VUV Undulator", PAC'07, Albuquerque, USA, 2007.

[3] X. R. Resende - LNLS - Internal communication.

[4] X. R. Resende "Estimation of Transverse Coupling from Pin-hole images” EPAC'06, 2006. 